\title{
Reactions of Pseudomonas aeruginosa pyocyanin with reduced glutathione
}

\author{
Rajkumar Cheluvappa ${ }^{1 凶}$, Ronald Shimmon², Michael Dawson², Sarah N. Hilmer ${ }^{1,3}$ \\ and David G. Le Couteur ${ }^{1}$ \\ ${ }^{1}$ Centre for Education and Research on Ageing and ANZAC Research Institute, University of Sydney and Con- \\ cord RG Hospital, Concord, Australia; ${ }^{2}$ Department of Chemistry, Material and Forensic Science, University \\ of Technology Sydney, Sydney, Australia; ${ }^{3}$ Departments of Aged Care and Clinical Pharmacology, Royal North \\ Shore Hospital, St Leonards, Australia
}

Received: 24 May, 2008; revised: 26 August, 2008; accepted: 16 September, 2008 available on-line: 17 September, 2008

\begin{abstract}
Pseudomonas aeruginosa is the most common cause of chronic and recurrent lung infections in patients with cystic fibrosis (CF) whose sputa contain copious quantities of $P$. aeruginosa toxin, pyocyanin. Pyocyanin triggers tissue damage mainly by its redox cycling and induction of reactive oxygen species (ROS). The reactions between reduced glutathione (GSH) and pyocyanin were observed using absorption spectra from spectrophotometry and the reaction products analysed by nuclear magnetic resonance imaging. Pyocyanin reacted with GSH non-enzymatically at $37^{\circ} \mathrm{C}$ resulting in the production of red-brown products, spectophotometrically visible as a 480 $\mathrm{nm}$ maximum absorption peak after $24 \mathrm{~h}$ of incubation. The reaction was concentration-dependent on reduced glutathione but not on pyocyanin. Minimizing the accessibility of oxygen to the reaction decreased its rate. The anti-oxidant enzyme catalase circumvented the reaction. ProtonNMR analysis demonstrated the persistence of the original aromatic ring and the methyl-group of pyocyanin in the red-brown products. Anti-oxidant agents having thiol groups produced similar spectophotometrically visible peaks. The presence of a previously unidentified non-enzymatic GSH-dependent metabolic pathway for pyocyanin has thus been identified. The reaction between pyocyanin and GSH is concentration-, time-, and $\mathrm{O}_{2}$-dependent. The formation of $\mathrm{H}_{2} \mathrm{O}_{2}$ as an intermediate and the thiol group in GSH seem to be important in this reaction.
\end{abstract}

Keywords: catalase, superoxide dismutase, glutathione, Pseudomonas aeruginosa pyocyanin, cystic fibrosis, oxidative stress, spectrophotometry, nuclear magnetic resonance imaging

\section{INTRODUCTION}

Chronic Pseudomonas aeruginosa infections occur frequently with the immunocompromised, the aged, and those with cystic fibrosis (CF) (Lyczak et al., 2002), bronchiectasis (Khalid et al., 2004), and otitis externa (Beers \& Abramo, 2004). Antibiotic-resistant $P$. aeruginosa infections are a major source of concern (Conway et al., 2003). P. aeruginosa secretes a number of virulence factors, including elastases, rhamnolipids, alginate, exotoxin A, exoenzyme S, and phospholipase $\mathrm{C}$, whose virulent properties have been elucidated well (Lyczak et al., 2000; Lau et al., 2004). Yet, neither the precise virulent properties, nor the in vivo metabolic fate of pyocyanin, a redox active blue-green phenazine pigment secreted

\footnotetext{
Corresponding author: Rajkumar Cheluvappa, Centre for Education and Research on Ageing, Concord RG Hospital, Hospital Road, Concord, Sydney, NSW 2139, Australia; phone: (61 02) 9767 8356; fax: (61 02) 9767 9101; e-mail: rcheluvappa@med.usyd.edu.au

Abbreviations: $\delta$, chemical shift; CF, cystic fibrosis; DTT, dithiothreitol; ELF, extracellular lung fluid; GSH, glutathione (reduced); GSSG, glutathione (oxidized); NAC, N-acetylcysteine; NMR, nuclear magnetic resonance imaging; ROS, reactive oxygen species.
} 
by $P$. aeruginosa in copious quantities in infected CF patients (Pitt, 1986; Wilson et al., 1988), have been charted adequately.

Sputum sol phase pyocyanin concentrations from patients with $\mathrm{CF}$ colonized by $P$. aeruginosa can go up to $27.3 \mu \mathrm{g} / \mathrm{ml}(\approx 130 \mu \mathrm{M})$ (Wilson et al., 1988). These concentrations are much more than adequate to inhibit ciliary beating as demonstrable in vitro (Wilson et al., 1987). Pyocyanin exerts diverse cytotoxic effects mediated mainly through its redox activity and reactive oxygen species (ROS)inducing capability (Mahajan-Miklos et al., 1999; O'Malley et al., 2004). Pyocyanin inhibits cell respiration (Sorensen \& Klinger, 1987), inactivates catalase (O'Malley et al., 2003), inhibits prostacyclin release (Kamath et al., 1995), and disrupts lung epithelial cell growth (Wilson et al., 1988), calcium homeostasis (Denning et al., 1998) and ciliary function (Wilson et al., 1987). Pyocyanin facilitates $P$. aeruginosa colonization of the respiratory tract by significantly reducing tracheobronchial mucus velocity (Munro et al., 1989). Pyocyanin inactivates protease inhibitors and contributes to a protease-antiprotease activity imbalance, resulting in lung epithelial injury (Britigan et al., 1999). Two in vivo murine respiratory tract infection models, acute and chronic, have demonstrated the absolute necessity of pyocyanin biosynthesis for P. aeruginosa virulence (Lau et al., 2004).

Glutathione (GSH), a ubiquitous tripeptide intracellular and extracellular thiol, constitutes $90 \%$ of intracellular nonprotein thiols (Meister \& Anderson, 1983). In a reaction catalyzed by GSH peroxidase (GPx), GSH converts hydrogen peroxide to water, and lipid peroxides to nontoxic hydroxy fatty acids (Zachara et al., 2006). In addition to this most important anti-oxidant function, GSH also impedes antiprotease-degradation (Buhl et al., 1990) and assists in maintaining lung surfactant (Jain et al., 1992). GSH levels in the extracellular lung fluid (ELF) range from 250 to $800 \mu \mathrm{M}$ with a mean of $429 \mu \mathrm{M}$ for patients not under oxidative stress, and a mean of $800 \mu \mathrm{M}$ for patients under oxidative stress (Roum et al., 1993; Morrison et al., 1999). This is significantly higher than the normal plasma GSH range of 2$5 \mu \mathrm{M}$ (Samiec et al., 1998). The total ELF glutathione ( $96 \%$ GSH $+4 \%$ glutathione disulfide GSSG) is at least 140 times more than the plasma concentration (Cantin et al., 1987). Although the ELF GSH levels are slightly lower in CF patients when compared to normal individuals (Roum et al., 1993), a normal level of intracellular GSH $(2-10 \mathrm{mM})$ is present in CF epithelial cells (Gao et al., 1999). In CF patients, both spontaneous and induced sputum samples contain higher GSH levels than samples from healthy controls (Dauletbaev et al., 2004).

Since pyocyanin is produced in large amounts in CF pseudomonal infections (Pitt, 1986; Wilson et al., 1988), these differences in GSH levels in the ELF and bronchoalveolar secretions could be easily and frequently perturbed by acute or chronic pyocyanininduced epithelial and endothelial damage, resulting in GSH release from the lysed cells. Pyocyanin (12.5 $\mu \mathrm{M}$ or more, $30 \mathrm{~min}$-incubation) significantly decreases human umbilical venous endothelial cell GSH level (Muller, 2002). Pyocyanin (30 $\mu \mathrm{M}$ or more, 24 hour-incubation) significantly decreases intracellular GSH in the respiratory cell-line A549 (O'Malley et al., 2004). Pyocyanin concentrations of $30 \mu \mathrm{M}$ or more significantly increase extracellular GSH export and GSSG concentrations in A549 cells (O'Malley et al., 2004). Those authors demonstrated a prompt direct reaction between pyocyanin and GSH, resulting in the formation of thiyl, pyocyanin, and superoxide radicals. Both Muller (2002) and O'Malley et al. (2004) reported the results of experiments involving pyocyanin and GSH only that were incubated for less than an hour. Yet in CF patients, P. aeruginosa, secreting pyocyanin, lingers for long periods of time causing recurrent remissions and exacerbations. We simulated this scenario by demonstrating a reaction between pathophysiologically relevant concentrations of pyocyanin and GSH over a period of days, rather than minutes or hours.

In our study, we show a reaction of pyocyanin with GSH in phosphate buffer ( $\mathrm{pH} 7.4$ ), at $37^{\circ} \mathrm{C}$, observed over a period of days under pathophysiologically relevant conditions using spectrophotometry. We also investigated the effect of oxygen concentration, reactant concentrations, anti-oxidant enzymes, and $\mathrm{pH}$ on the reaction. We also report our attempts to purify and analyze the reaction products using nuclear magnetic resonance (NMR) imaging.

\section{METHODS}

Synthesis of pyocyanin. Pyocyanin was chemically synthesized by photolysis of phenazine methosulfate (Knight et al., 1979) and purified (Muller \& Sorrell, 1992) as described earlier. Briefly, phenazine methosulfate (P-9625-5g, Sigma-Aldrich Pt Ltd, Sydney, Australia) was made up in $0.01 \mathrm{M} \mathrm{Tris} / \mathrm{HCl}$ buffer ( $\mathrm{pH}$ 7.4) in a round-bottomed Pyrex flask and exposed to fluorescent tube light (Phillips TLD 18 $\mathrm{W} / 54$ ) for $2.5 \mathrm{~h}$. The resulting solution was extracted into chloroform and vacuum-dried to a powdery residue. The residue was dissolved in chloroform and acidified with an equal volume of $0.1 \mathrm{M} \mathrm{HCl}$. The red acidic form of pyocyanin was converted into the blue form using $0.5 \mathrm{M} \mathrm{NaOH}$. This blue form of pyocyanin, now in the aqueous phase, was extracted into chloroform and this cycle repeated 3 times. The blue form of pyocyanin which was extracted into chloroform the last time was vacuum dried, washed 
as well as precipitated with hexane, trapped on a type $\mathrm{EH} 0.5 \mu \mathrm{m}$ filter, reconstituted in chloroform and purified by thin-layer chromatography (TLC) using an equimolar chloroform/methanol mixture solvent. The pyocyanin thus purified was stored in methanol at $-70^{\circ} \mathrm{C}$. The purity of pyocyanin was ascertained and its concentration quantitated by utilizing its known absorption spectrum and absorption coefficient values as elucidated earlier (Watson et al., 1986). Before use, the methanol solvent was removed by nitrogen insufflation and when completely dry, pyocyanin was reconstituted in saline and used.

Glutathione-pyocyanin reactions. All reactions were done in triplicate. Pyocyanin concentrations used were $0-125 \mu \mathrm{M}$ to cover previously estimated in vivo concentrations (Wilson et al., 1988). GSH concentrations utilized were $0-10 \mathrm{mM}$ to cover concentration ranges in CF ELF (Roum et al., 1993), CF epithelial cells (Gao et al., 1999) and in spontaneous and induced sputa (Dauletbaev et al., 2004). Most reactions had $25 \mu \mathrm{M}$ pyocyanin and $10 \mathrm{mM}$ GSH as the reactants. All incubation mixtures were incubated in well-covered $1 \mathrm{~cm}$ plastic cuvettes, unless otherwise specified. Incubation of all reactions was done in phoshate buffer solution (PBS, $125 \mathrm{mM}$ $\mathrm{KH}_{2} \mathrm{PO}_{4}, \mathrm{pH} 7.4$ ) at $37^{\circ} \mathrm{C}$ in the dark. For oxygendeprived reactions, nitrogen was bubbled through the reaction mixtures, followed by addition of a 3 $\mathrm{cm}$ mineral oil layer on top of each final reaction mixture before incubation. Reaction mixtures were transferred to plastic cuvettes to measure the absorbance spectra using a Shimadzu UV120 IPC spectrophotometer with the following calibrations: Measuring Mode - Abs, Recording Range - Low 0.0 to High 0.5, Wavelength Range (nm) - 800-200 $\mathrm{nm}$, Scan Speed - Fast and Sampling Interval - 1 $\mathrm{nm}$. The absorption spectra presented throughout this study are representatives of triplicates. The reaction rates and constants were deliberately not calculated owing to the obvious slow reaction time and the chronicity of the pathophysiological scenario.

Purification of glutathione-pyocyanin reaction products. The red-brown GSH-pyocyanin reaction products were purified from up-scaled $10 \mathrm{ml}$ reaction mixtures (concentrations of reactants increased 10-fold) with the discovery that a Tris/ $\mathrm{HCl}$ $(10 \mathrm{mM}, \mathrm{pH} 3)$ wash prior to acidified (2\% acetic acid) aqueous methanol elution of the GSH-pyocyanin reaction products removes glutathione, both reduced and oxidized. The presence or absence of glutathione is easily discernable by the presence or absence of the $230 \mathrm{~nm}$ peak or by the ninhydrin test. Each up-scaled reaction mixture consisted of $10 \mathrm{ml}$ of PBS with $250 \mu \mathrm{M}$ pyocyanin and $100 \mathrm{mM}$ GSH (final $\mathrm{pH}=7.4$ ), incubated at $37^{\circ} \mathrm{C}$ in a loosely capped $50 \mathrm{ml}$ conical bottom Falcon polypropyl- ene tube for $36-48 \mathrm{~h}$. After several attempts, purification of the red-brown species from the reactant remnants and other products involved the following solid phase extraction (SPE) method: A polymer based SPE column (Oasis HLB 3cc cartridges from Waters company) was determined to be the best available method to purify the red-brown species by utilizing the following protocol (for each Oasis HLB 3cc cartridge): (1) $\mathrm{CH}_{3} \mathrm{OH}: 1 \mathrm{ml}$, (2) Millipore water: $1 \mathrm{ml}$, (3) up-scaled pyocyanin-GSH reaction mixture: $1 \mathrm{ml},(4)$ Tris/ $\mathrm{HCl}$ wash: $1 \mathrm{ml}$, (5) PBS ( $\mathrm{pH}=7.4$ ) wash: $5 \mathrm{ml}$, (6) Millipore water: $1 \mathrm{ml}$, (7) acidified $60 \%$ methanol elute solution: $1 \mathrm{ml}$, and (8) speed vacuum drying.

Ninhydrin test. The ninhydrin test is a test for the presence or absence of the amino $\left(-\mathrm{NH}_{2}\right)$ group and hence should be positive if glutathione (GSH or GSSG) is present. A crude qualitative visual test that we utilized for testing for the presence or absence of glutathione was done by heating $0.5 \mathrm{ml}$ of the sample with $0.5 \mathrm{ml}$ of ninhydrin (triketohydrindene hydrate; Sigma-Aldrich, St. Louis, MO, USA) in acetone with $1 \%$ acetic acid up to $45^{\circ} \mathrm{C}$ in a glass tube and observing color changes. In this adaptation of the ninhydrin test, a dark red-brown color change indicates the presence of abundant amino $\left(-\mathrm{NH}_{2}\right)$ groups in the sample. This crude test was positive only when amino groups were abundant.

Chemicals and enzymes. Glutathione (GSH and GSSG), N-acetylcysteine (NAC), dithiothreitol (DTT), superoxide dismutase (SOD), and catalase (CAT) were purchased from Sigma-Aldrich (St. Louis, MO, USA). TLC plates (Silicagel $20 \times 20 \mathrm{~cm}$ ) were obtained from Crown Scientific (Moorebank, NSW, Australia).

Nuclear magnetic resonance imaging. All proton NMR $\left({ }^{1} \mathrm{H}\right.$ solution) spectra were done at $25^{\circ} \mathrm{C}$ using a Bruker DRX $300 \mathrm{MHz}$ NMR instrument. Deuterium oxide (Sigma Aldrich) was consistently used as the solvent. The internal standard was the sodium salt of 3-(trimethylsilyl) 3,3,2,2-tetradeuteroproponic acid. The typical acquisition parameters utilized were spectral width $4000 \mathrm{~Hz}$, repetition time $2 \mathrm{~s}$ and line broadening frequency $0.5 \mathrm{~Hz}$.

\section{RESULTS}

\section{Glutathione and pyocyanin reaction}

GSH reacts with pyocyanin non-enzymatically after $24 \mathrm{~h}$ of incubation at $37^{\circ} \mathrm{C}$, resulting in formation of red-brown products, visible spectophotometrically as a $480 \mathrm{~nm}$ peak (Fig. 1E and $\mathrm{G})$. Figure $1 \mathrm{~A}$ and $\mathrm{B}$ represent $10 \mathrm{mM}$ GSH-only controls under aerobic and oxygen-deprived con- 
ditions, respectively. Figures $1 \mathrm{C}$ and $\mathrm{D}$ represent $25 \mu \mathrm{M}$ pyocyanin-only controls under aerobic and oxygen-deprived conditions, respectively.

Time-course experiments involving glutathione and pyocyanin

GSH and pyocyanin were incubated at $37^{\circ} \mathrm{C}$ over $96 \mathrm{~h}$ under normal aerobic conditions or oxygen-deprived conditions, with absorption spectra recorded 24-hourly. The production of the $480 \mathrm{~nm}$ peaks, coinciding with the formation of the visible red-brown products, was much faster under aero-
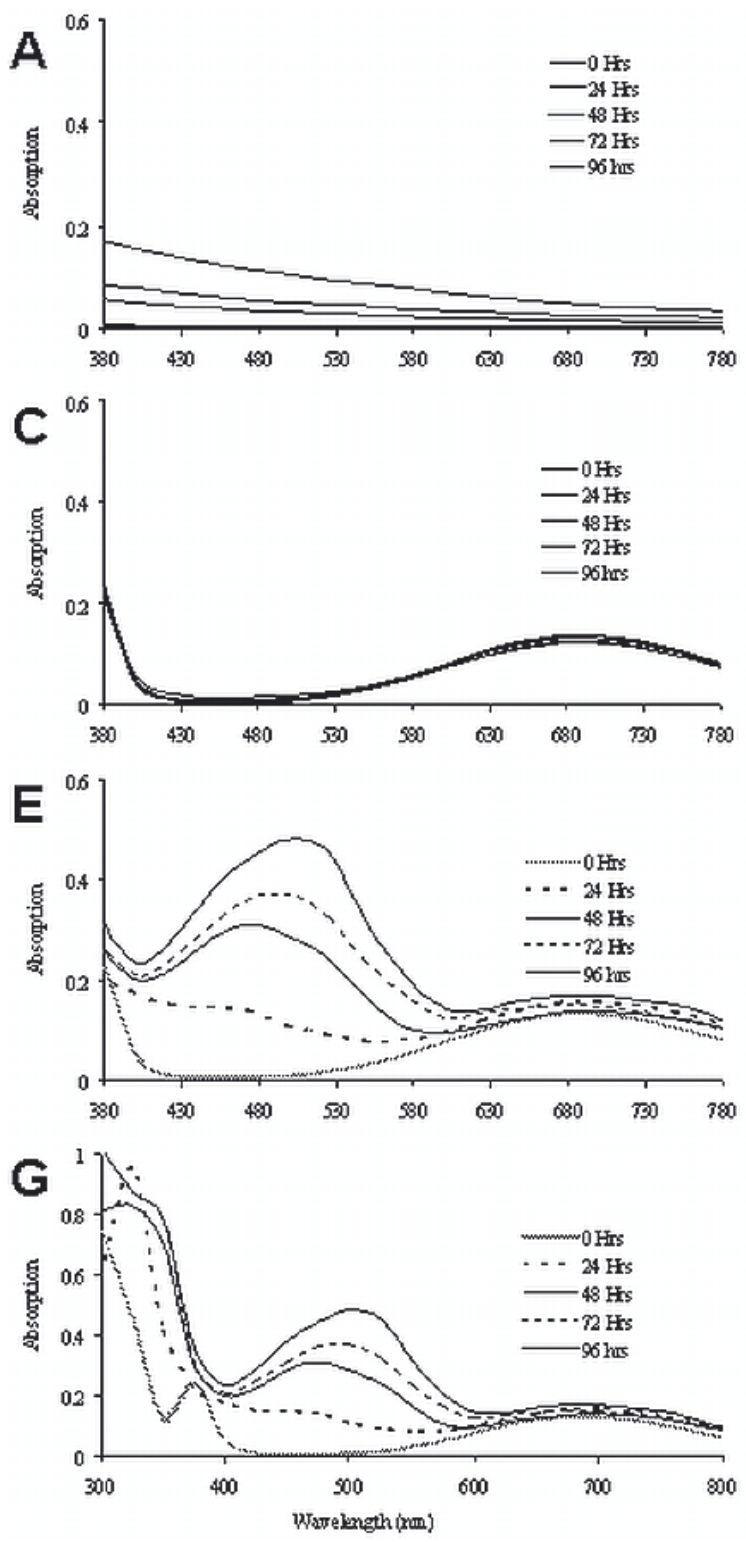

bic conditions (Figs. 1E and G) in contrast to oxygen-deprived conditions (Fig. $1 \mathrm{~F}$ and $\mathrm{H}$ ).

\section{Dose-response experiments involving glutathione and pyocyanin}

Dose-response experiments were done involving GSH and pyocyanin at $37^{\circ} \mathrm{C}$ over $96 \mathrm{~h}$ under normal aerobic conditions. Production of the $480 \mathrm{~nm}$ peak, coinciding with the formation of the visible red-brown products, was dependent only on the concentration of GSH (Fig. 2A) but not on the concentration of pyocyanin (Fig. 2B).
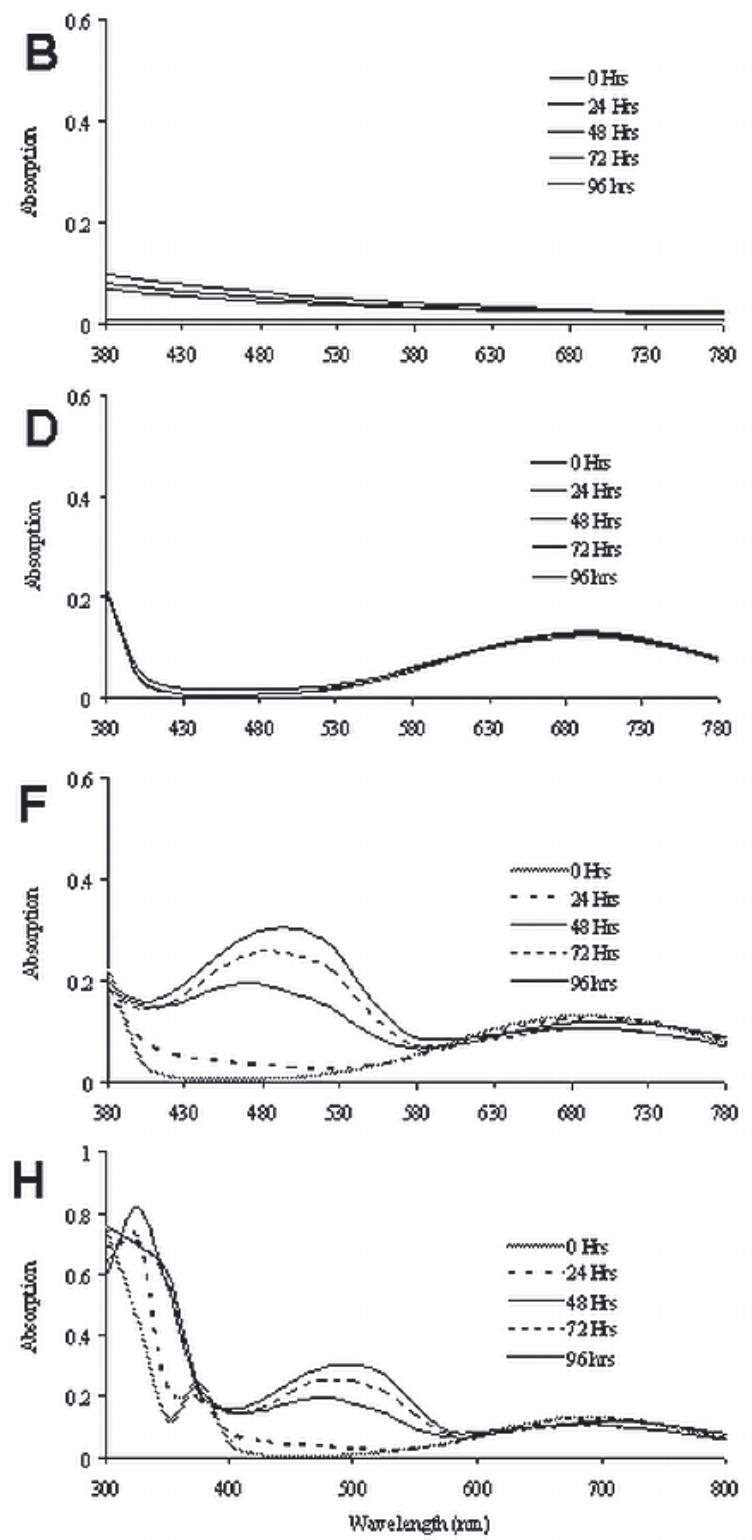

Figure 1. Time-course experiments involving GSH and pyocyanin.

GSH and pyocyanin were allowed to react at $37^{\circ} \mathrm{C}$ over $96 \mathrm{~h}$ with absorption spectra recorded 24-hourly. A and B, 10 mM GSH-only controls under aerobic and oxygen-deprived conditions, respectively. C and D, $25 \mu \mathrm{M}$ pyocyanin-only controls under aerobic and oxygen-deprived conditions, respectively. E and F, $25 \mu \mathrm{M}$ of pyocyanin reacting with $10 \mathrm{mM}$ GSH under aerobic and oxygen-deprived conditions, respectively, demonstrating that the reaction producing the $480 \mathrm{~nm}$ absorption peak was much faster under aerobic conditions than anaerobic conditions. $\mathrm{G}$ and $\mathrm{H}$, Complete spectra shown in part as $\mathrm{E}$ and $\mathrm{F}$, respectively. 

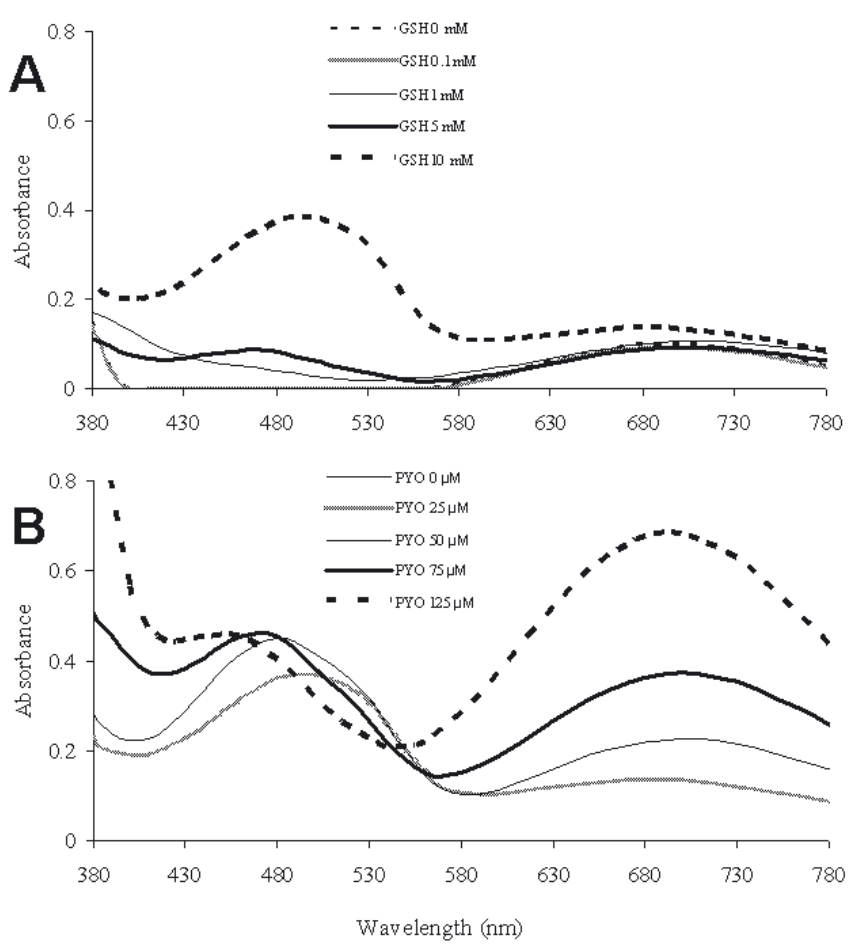

Figure 2. Dose-response experiments involving GSH and pyocyanin.

GSH and pyocyanin were allowed to react at $37^{\circ} \mathrm{C}$ over $96 \mathrm{~h}$ under aerobic conditions. A. Absorption spectra for 25 $\mu \mathrm{M}$ pyocyanin treated with increasing concentrations of GSH. Production of the $480 \mathrm{~nm}$ peak was dependent on the concentration of GSH. B. Absorption spectra for $10 \mathrm{mM}$ GSH treated with increasing concentrations of pyocyanin. Production of the $480 \mathrm{~nm}$ peak did not seem to be dependent on the concentration of pyocyanin.
The effect of anti-oxidant enzymes on the glutathione-pyocyanin reaction and the effect of $\mathrm{pH}$ variations on the reaction products

SOD did not prevent the 72-h GSH-pyocyanin reaction from occurring but a combination of SOD and catalase did (Fig. 3A), pointing to the importance of $\mathrm{H}_{2} \mathrm{O}_{2}$ but not superoxide in the reaction. A completely and repeatedly demonstrable $\mathrm{pH}$-dependant ( $\mathrm{pH} 2$ to 9) reversible shift in the complete absorption spectra of a diluted sample of the redbrown GSH-pyocyanin reaction products was evi- dent (Fig. 3B), suggesting active electron resonance in the reaction products.

\section{Purification of glutathione-pyocyanin reaction prod-} ucts

Solid-phase extraction procedures involving various concentrations, proportions and combinations of methanol, chloroform, ethyl acetate and acetone were done before ascertaining that acidified methanol was the best eluant for solid-phase-trapped red-brown GSH-pyocyanin reaction products. The more the aque-
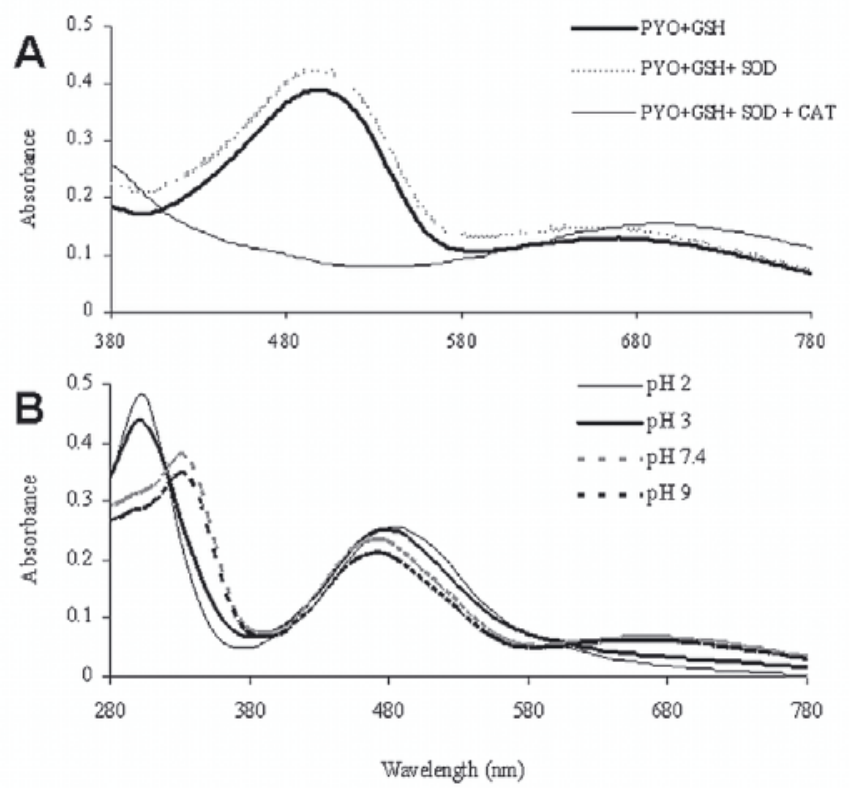

Figure 3. The effect of anti-oxidant enzymes on the GSH-pyocyanin reaction and the effect of $\mathrm{pH}$ variations on the reaction products.

A. SOD did not prevent the 72-h GSH-pyocyanin reaction from occurring but a combination of SOD and catalase did. B. pHdependant reversible shift ( $\mathrm{pH} 2$ to 9) in absorption spectra of diluted sample of the red-brown GSH-pyocyanin reaction products. 

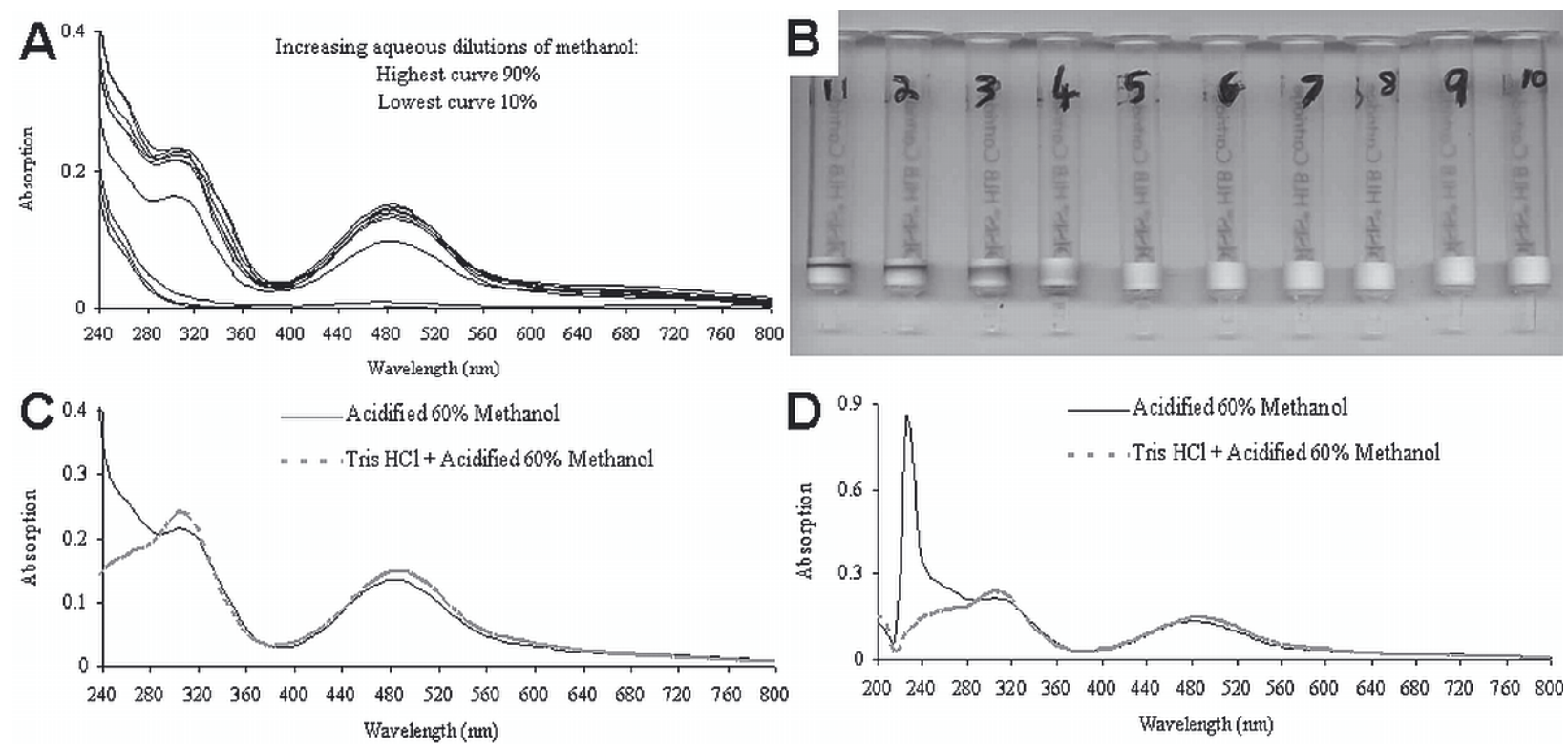

Figure 4. Purification of GSH-pyocyanin reaction products.

$\mathrm{A}$ and B. Demonstration of the efficacy of increasing dilutions of aqueous acidified methanol to elute the red-brown GSH-pyocyanin reaction products trapped in the Oasis HLB 3cc cartridges. B. Cartridges 1 to 10 arranged in order of increasing dilutions of acidified methanol demonstrating that more the aqueous dilution of acidified methanol, the better the elution efficacy of the trapped red-brown GSH-pyocyanin reaction products. However, owing to the ease in extracting the dry GSH-pyocyanin reaction products from $60 \%$ methanol compared to more aqueous solutions, this dilution of acidified methanol was used subsequently. C and D. Spectra of $60 \%$ acidified methanol eluted GSH-pyocyanin reaction products with or without Tris/ $\mathrm{HCl}(10 \mathrm{mM}, \mathrm{pH} 3)$ removal of residual glutathione. Absence of residual glutathione was confirmed by the absence of the $230 \mathrm{~nm}$ absorption peak and a negative ninhydrin test. D. Complete spectra shown in part as C.

ous dilution of acidified methanol ( $2 \%$ acetic acid), the better the elution efficacy of the trapped red-brown GSH-pyocyanin reaction products trapped in the Oasis HLB 3cc cartridges (Figs. 4A and B). Having estab- lished that acidified $60 \%$ methanol was the most appropriate eluant (Figs. 4A and B) owing to the ease in extracting the dry reaction products from $60 \%$ methanol compared to more aqueous solutions, the problem

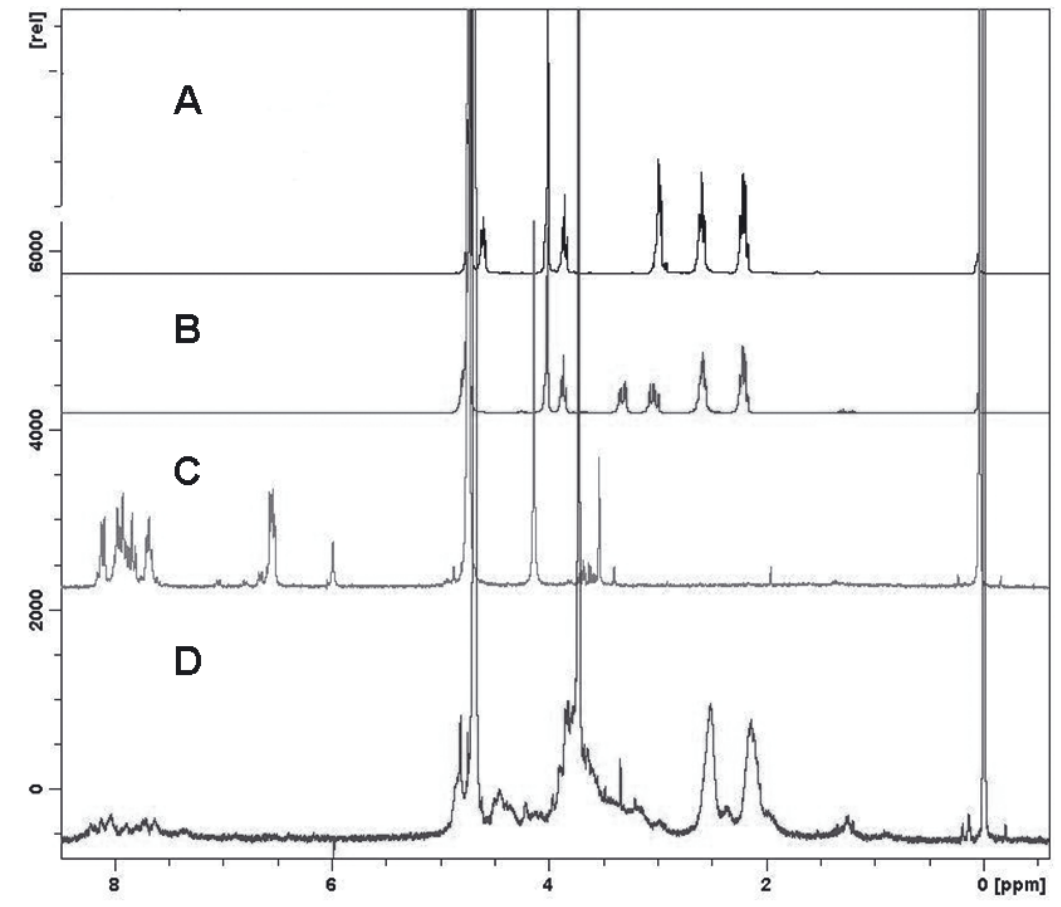

Figure 5. Proton NMR analysis of the red-brown GSH-pyocyanin reaction products.

Proton NMR spectra of GSH (A), GSSG (B), Pyocyanin (C) and red-brown GSH-pyocyanin reaction products (D). The singlet peak at $\delta 4.14 \mathrm{ppm}$, corresponding to the methyl group in pyocyanin (C), shifts upfield in the redbrown GSH-pyocyanin reaction products (D) with additional peaks in the latter $(\delta$ 2.14, 2.53, 3.82, 4.21 and 4.81) corresponding to GS in the red-brown GSH-pyocyanin reaction products. The multiple peaks around $\delta 7.64-8.21$ $\mathrm{ppm}$, indicative of the aromatic region of the pyocyanin (C), shifted downfield in the red-brown GSH-pyocyanin reaction products (D). 


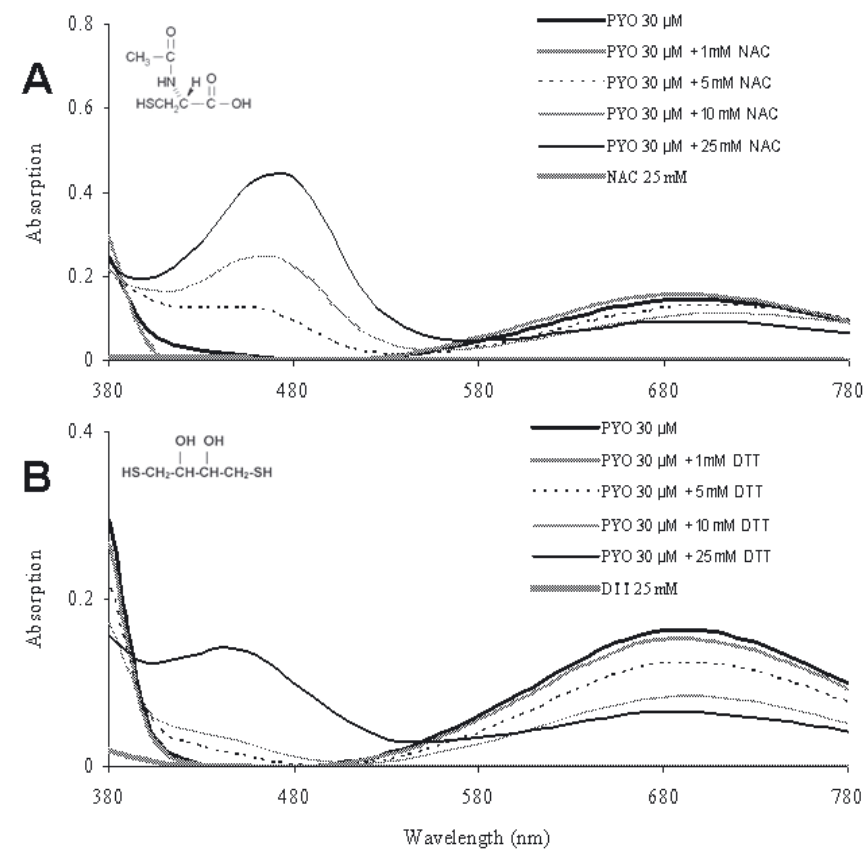

Figure 6. Reactions of pyocyanin with other thiol anti-oxidant agents

At $37^{\circ} \mathrm{C}$ over $96 \mathrm{~h}$ under aerobic conditions, thiol anti-oxidant agents $\mathrm{N}$-acetylcysteine (A) and dithiothreitol (B) produced absorption peaks at $470 \mathrm{~nm}$ and $450 \mathrm{~nm}$ respectively, with $30 \mu \mathrm{M}$ pyocyanin. of co-elution of the original reactants (both reduced and oxidized glutathione) with the products was precluded only after the discovery that Tris/ $\mathrm{HCl}(10 \mathrm{mM}$, $\mathrm{pH} 3$ ) removes glutathione (GSH and GSSG). The absence of residual glutathione can be inferred from the absence of the $230 \mathrm{~nm}$ absorption peak (Figs. 4A and $4 \mathrm{D}$; Fig. $4 \mathrm{C}$ is a magnified circumscribed version of Fig. 4D), after Tris/HCl treatment and subsequent acidified aqueous methanol elution of the GSH-pyocyanin reaction red-brown products. These products were also ninhydrin test-negative suggesting the absence of abundant amino group and hence, glutathione.

\section{Properties of glutathione-pyocyanin reaction products}

The purified red-brown products were insoluble in methanol, chloroform, ethyl acetate, acetone, and a number of organic solvents, but very soluble in water.

Nuclear magnetic resonance analysis of the redbrown glutathione-pyocyanin reaction products

A comparison of the proton NMR spectra of GSH (Fig. 5A), GSSG (Fig. 5B), pyocyanin (Fig. 5C) and the red-brown GSH-pyocyanin reaction products (Fig. 5D) provides an indication of the appropriate assignments to the red-brown GSH-pyocyanin reaction products. The singlet peak at $\delta 4.14 \mathrm{ppm}$, which corresponds to the methyl group in pyocyanin (Fig. 5C), shifts upfield in the red-brown GSH-pyocyanin reaction products (Fig. 5D) due to the disappearance of the positive charge from the amine group. In the NMR spectra of the red-brown GSH-pyocyanin reaction products (Fig. 5D), five peaks at $\delta 2.14,2.53,3.82,4.21$ and 4.81 can be assigned to the GS bond to the pyocyanin owing to similar peaks produced by GSH and GSSG. Furthermore, the multiple peaks around $\delta$ 7.64-8.21 ppm, indicative of the aromatic region of the pyocyanin (Fig. 5C), shifted downfield in the red-brown GSH-pyocyanin reaction products (Fig. 5D). This downfield shift in the aromatic region of the NMR spectra may be the characteristic feature of the new net positive charge of the red-brown GSH-pyocyanin reaction products.

\section{Reactions of pyocyanin with other thiol anti-oxidant agents}

Thiol anti-oxidant agents $N$-acetylcysteine (Fig. 6A) and dithiothreitol (Fig. 6B) produced ab-

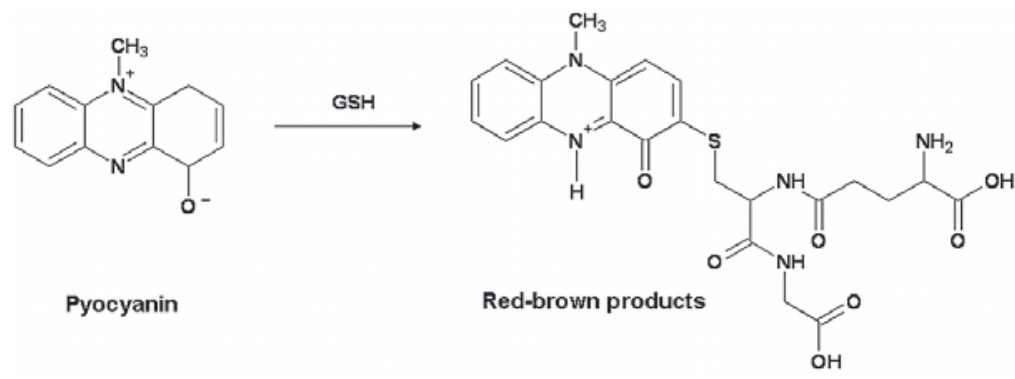

Figure 7. Proposed reaction of pyocyanin with GSH.

Hydrogen from the thiol (-SH) group of GSH reacts with the first nitrogen atom of pyocyanin. The hydroquinone pyocyanin structure is oxidized to a quinone structure, adding a whole GS molecule to the ortho-position of the quinone red-brown products, with a re-arranging of covalent carbon bonds. 
sorption peaks with $30 \mu \mathrm{M}$ pyocyanin $\left(37^{\circ} \mathrm{C} ; 96 \mathrm{~h}\right.$; normal aerobic conditions) at $470 \mathrm{~nm}$ and $450 \mathrm{~nm}$ respectively, demonstrating that the thiol group may be important in these reactions.

\section{DISCUSSION}

Pyocyanin reacted non-enzymatically with GSH resulting in formation of red-brown products, visible spectophotometrically as a $480 \mathrm{~nm}$ peak recorded after $24 \mathrm{~h}$ of incubation at $37^{\circ} \mathrm{C}$. The reaction was concentration-dependent on GSH. Decreasing oxygen availability decelerated the reaction. The redbrown products also demonstrated an absorption spectral shift with $\mathrm{pH}$ changes. The red-brown products were insoluble in methanol, chloroform, ethyl acetate, acetone, and a number of organic solvents, but very soluble in water. Catalase circumvented the reaction demonstrating the importance of $\mathrm{H}_{2} \mathrm{O}_{2}$ in this reaction. Redox cycling of pyocyanin utilizing cellular reductants results in the production of abundant quantities of $\mathrm{H}_{2} \mathrm{O}_{2}$ which can be used in this reaction (Muller, 2002). Thiol group-containing agents such as NAC and DTT produced similar reactions showing that the thiol group may be important in this reaction. Proton-NMR analysis clearly depicted the persistence of the original aromatic ring and the methyl group of pyocyanin in the red-brown products. Hydrogen from the thiol group of GSH or a possible thiyl radical that may have formed in the reaction between pyocyanin and GSH (O'Malley et al., 2004) may react with the first nitrogen atom of pyocyanin (Fig. 7). GSH probably reacts with the hydroquinone pyocyanin structure producing a quinone structure, resulting in a whole GS molecule being added to the ortho-position of the quinone redbrown products in the process.

Pyocyanin, which is produced in large amounts in CF pseudomonal infections (Pitt, 1986; Wilson et al., 1988), exerts manifold cytotoxic effects mediated through redox cycling and ROS induction (Britigan et al., 1992; Mahajan-Miklos et al., 1999; Muller, 2002). Pyocyanin alters specific immune defences, and potentiates and perpetuates harmful inflammatory reactions in the infected CF lung. Yet the in vivo metabolic fate of pyocyanin has not been elucidated till now.

Lung epithelial, endothelial, and ELF GSH exerts significant cytoprotective antioxidant, surfactant stabilizing, anti-protease protecting and mucolytic activity (Meister \& Anderson, 1983; Rahman \& MacNee, 2000). Abnormal and low GSH levels can lead to crippling of the lung's antioxidant system, antiprotease system (Konstan et al., 1994; Birrer, 1995), message transmission and immune recognition system (Barbero, 1994), mucolytic system (Hudson,
2001; 2004), and surfactant secretion system (Jain et al., 1992). The end results of low GSH levels include pulmonary damage, exacerbation of extant pseudomonal infections and a drastically increased propensity to contract infections. Since pyocyanin has a profound ability to modulate the glutathione redox cycle and deplete intracellular GSH in endothelial cells (Muller, 2002) and intracellular and extracellular GSH in lung epithelial cells (O'Malley et al., 2004), it is reasonable to expect lower GSH levels in ELF from CF patients with $P$. aeruginosa infection. Although ELF has decreased GSH levels (Gao et al., 1999) compared to intracellular GSH levels in CF lung epithelia, CF sputum samples have higher GSH levels than healthy sputum samples (Dauletbaev et al., 2004). The latter discrepancies could be accentuated by pyocyanin-induced epithelial (Hingley et al., 1986; Wilson et al., 1987) and endothelial (Britigan et al., 1992; Miller et al., 1996; Muller, 2002) necrosis and apoptosis, resulting in GSH release. Since normal intracellular GSH concentration ranges from 2 to $10 \mathrm{mM}$ (Gao et al., 1999), released cellular GSH would probably be present in high concentrations for a non-enzymatic reaction with pyocyanin in the setting of a persistent $P$. aeruginosa infection lingering in the CF lung.

While pyocyanin is very soluble in water, methanol and chloroform, the purified red-brown products were insoluble in most organic solvents. Yet the red-brown products were very soluble in water, attesting to the polarity of the same and suggesting that this reaction is probably a pyocyanin detoxification mechanism. Preliminary studies with NADPH show a significant drop in the oxidative potential of the red-brown products when compared to that of pyocyanin. Therefore the red-brown products may be the result of a previously unknown oxygendependent, non-enzymatic pyocyanin detoxification system involving GSH. Consequentially, the production of pyocyanin in the airways of CF patients may be much higher than previously assessed (Pitt, 1986; Wilson et al., 1988), because the presence of the red-brown products has not been accounted for by the methodologies used till now. Neither the redbrown products produced during this reaction, nor the magnitude of GSH concentration fluctuations in CF patients with $P$. aeruginosa infections, have been estimated yet. This can be the next research course to pursue.

To conclude, the presence of a possible nonenzymatic GSH-dependent pathway for $P$. aeruginosa pyocyanin conversion has been identified. This pathway is time-, concentration-, and $\mathrm{O}_{2}$-dependent. The formation of $\mathrm{H}_{2} \mathrm{O}_{2}$ as an intermediate is necessary for this reaction. The thiol group of GSH seems to be important in this reaction. The original aromatic ring and the methyl group of pyocyanin persist in 
the red-brown reaction products. Strategies to inhibit pyocyanin production and to facilitate pyocyanin metabolism to neutralize its toxicity by GSH and other antioxidant therapies may be incorporated into existing regimens for the treatment of CF (Conway, 1999; Roum et al., 1999; Rahman \& MacNee, 2000). If the red-brown products prove to be substantially less pathogenic than pyocyanin in future research, the utilization of aerosolized, spaced, or nebulized GSH could be viable therapeutic options in chronic pseudomonal lung infections.

\section{Acknowledgements}

We would like to thank Dr. Michael Muller for all of his suggestions and guidance in this project. We would like to thank Professor Robin Fraser, Department of Pathology, Christchurch School of Medicine, University of Otago (Christchurch, New Zealand), for his continued guidance and inspiration.

This work was supported by the Ageing and Alzheimer's Research Foundation.

\section{REFERENCES}

Barbero GJ (1994) Adverse life events and exacerbations of cystic fibrosis. Pediatr Pulmonol 18: 73-76.

Beers SL, Abramo TJ (2004) Otitis externa review. Pediatr Emerg Care 20: 250-256.

Birrer P (1995) Proteases and antiproteases in cystic fibrosis: pathogenetic considerations and therapeutic strategies. Respiration 62 (Suppl 1): 25-28.

Britigan BE, Roeder TL, Rasmussen GT, Shasby DM, McCormick ML, Cox CD (1992) Interaction of the Pseudomonas aeruginosa secretory products pyocyanin and pyochelin generates hydroxyl radical and causes synergistic damage to endothelial cells. Implications for Pseudomonas-associated tissue injury. J Clin Invest 90: 2187-2196.

Britigan BE, Railsback MA, Cox CD (1999) The Pseudomonas aeruginosa secretory product pyocyanin inactivates alpha1 protease inhibitor: implications for the pathogenesis of cystic fibrosis lung disease. Infect Immun 67: 1207-1212.

Buhl R, Vogelmeier C, Critenden M, Hubbard RC, Hoyt RF Jr, Wilson EM, Cantin AM, Crystal RG (1990) Augmentation of glutathione in the fluid lining the epithelium of the lower respiratory tract by directly administering glutathione aerosol. Proc Natl Acad Sci USA 87: 4063-4067.

Cantin AM, North SL, Hubbard RC, Crystal RG (1987) Normal alveolar epithelial lining fluid contains high levels of glutathione. J Appl Physiol 63: 152-157.

Conway SP (1999) Evidence for using nebulised antibiotics in cystic fibrosis. Arch Dis Child 80: 307-309.

Conway SP, Brownlee KG, Denton M, Peckham DG (2003) Antibiotic treatment of multidrug-resistant organisms in cystic fibrosis. Am J Respir Med 2: 321-332.

Dauletbaev N, Viel K, Buhl R, Wagner TO, Bargon J (2004) Glutathione and glutathione peroxidase in sputum samples of adult patients with cystic fibrosis. J Cyst Fibros 3: 119-124.
Denning GM, Railsback MA, Rasmussen GT, Cox CD, Britigan BE (1998) Pseudomonas pyocyanine alters calcium signaling in human airway epithelial cells. Am J Physiol 274: L893-L900.

Gao L, Kim KJ, Yankaskas JR, Forman HJ (1999) Abnormal glutathione transport in cystic fibrosis airway epithelia. Am J Physiol 277: L113-L118.

Hingley ST, Hastie AT, Kueppers F, Higgins ML, Weinbaum G, Shryock T (1986) Effect of ciliostatic factors from Pseudomonas aeruginosa on rabbit respiratory cilia. Infect Immun 51: 254-262.

Hudson VM (2001) Rethinking cystic fibrosis pathology: the critical role of abnormal reduced glutathione (GSH) transport caused by CFTR mutation. Free Radic Biol Med 30: 1440-1461.

Hudson VM (2004) New insights into the pathogenesis of cystic fibrosis: pivotal role of glutathione system dysfunction and implications for therapy. Treat Respir Med 3: 353-363.

Jain A, Martensson J, Mehta T, Krauss AN, Auld PA, Meister A (1992) Ascorbic acid prevents oxidative stress in glutathione-deficient mice: effects on lung type 2 cell lamellar bodies, lung surfactant, and skeletal muscle. Proc Natl Acad Sci USA 89: 5093-5097.

Kamath JM, Britigan BE, Cox CD, Shasby DM (1995) Pyocyanin from Pseudomonas aeruginosa inhibits prostacyclin release from endothelial cells. Infect Immun 63: 4921-4923.

Khalid M, Saleemi S, Zeitouni M, Al Dammas S, Khaliq MR (2004) Effect of obstructive airway disease in patients with non-cystic fibrosis bronchiectasis. Ann Saudi Med 24: 284-287.

Knight M, Hartman PE, Hartman Z, Young VM (1979) A new method of preparation of pyocyanin and demonstration of an unusual bacterial sensitivity. Anal Biochem 95: 19-23.

Konstan MW, Hilliard KA, Norvell TM, Berger M (1994) Bronchoalveolar lavage findings in cystic fibrosis patients with stable, clinically mild lung disease suggest ongoing infection and inflammation. Am J Respir Crit Care Med 150: 448-454.

Lau GW, Hassett DJ, Ran H, Kong F (2004a) The role of pyocyanin in Pseudomonas aeruginosa infection. Trends Mol Med 10: 599-606.

Lau GW, Ran H, Kong F, Hassett DJ, Mavrodi D (2004b) Pseudomonas aeruginosa pyocyanin is critical for lung infection in mice. Infect Immun 72: 4275-4278.

Lyczak JB, Cannon CL, Pier GB (2000) Establishment of Pseudomonas aeruginosa infection: lessons from a versatile opportunist. Microbes Infect 2: 1051-1060.

Lyczak JB, Cannon CL, Pier GB (2002) Lung infections associated with cystic fibrosis. Clin Microbiol Rev 15: 194-222.

Mahajan-Miklos S, Tan MW, Rahme LG, Ausubel FM (1999) Molecular mechanisms of bacterial virulence elucidated using a Pseudomonas aeruginosa-Caenorhabditis elegans pathogenesis model. Cell 96: 47-56.

Meister A, Anderson ME (1983) Glutathione. Annu Rev Biochem 52: 711-760.

Miller RA, Rasmussen GT, Cox CD, Britigan BE (1996) Protease cleavage of iron-transferrin augments pyocyanin-mediated endothelial cell injury via promotion of hydroxyl radical formation. Infect Immun 64: 182-188.

Morrison D, Rahman I, Lannan S, MacNee W (1999) Epithelial permeability, inflammation, and oxidant stress in the air spaces of smokers. Am J Respir Crit Care Med 159: 473-479. 
Muller M (2002) Pyocyanin induces oxidative stress in human endothelial cells and modulates the glutathione redox cycle. Free Rad Biol Med 33: 1527-1533.

Muller M, Sorrell TC (1992) Leukotriene B4 omega-oxidation by human polymorphonuclear leukocytes is inhibited by pyocyanin, a phenazine derivative produced by Pseudomonas aeruginosa. Infect Immun 60: 2536-2540.

Munro NC, Barker A, Rutman A, Taylor G, Watson D, McDonald-Gibson WJ, Towart R, Taylor WA, Wilson R, Cole PJ (1989) Effect of pyocyanin and 1-hydroxyphenazine on in vivo tracheal mucus velocity. I Appl Physiol 67: 316-323.

O'Malley YQ, Reszka KJ, Rasmussen GT, Abdalla MY, Denning GM, Britigan BE (2003) The Pseudomonas secretory product pyocyanin inhibits catalase activity in human lung epithelial cells. Am J Physiol Lung Cell Mol Physiol 285: L1077-L1086.

O'Malley YQ, Reszka KJ, Spitz DR, Denning GM, Britigan BE (2004) Pseudomonas aeruginosa pyocyanin directly oxidizes glutathione and decreases its levels in airway epithelial cells. Am J Physiol Lung Cell Mol Physiol 287: L94-L103.

Pitt TL (1986) Biology of Pseudomonas aeruginosa in relation to pulmonary infection in cystic fibrosis. $J R$ Soc Med 79 (Suppl 12): 13-18.

Rahman I, MacNee W (2000) Oxidative stress and regulation of glutathione in lung inflammation. Eur Respir J 16: 534-554.

Roum JH, Buhl R, McElvaney NG, Borok Z, Crystal RG (1993) Systemic deficiency of glutathione in cystic fibrosis. J Appl Physiol 75: 2419-2424.
Roum JH, Borok Z, McElvaney NG, Grimes GJ, Bokser AD, Buhl R, Crystal RG (1999) Glutathione aerosol suppresses lung epithelial surface inflammatory cellderived oxidants in cystic fibrosis. J Appl Physiol 87: 438-443.

Samiec PS, Drews-Botsch C, Flagg EW, Kurtz JC, Sternberg P Jr, Reed RL, Jones DP (1998) Glutathione in human plasma: decline in association with aging, age-related macular degeneration, and diabetes. Free Radic Biol Med 24: 699-704.

Sorensen RU, Klinger JD (1987) Biological effects of Pseudomonas aeruginosa phenazine pigments. Antibiot Chemother 39: 113-124.

Watson D, MacDermot J, Wilson R, Cole PJ, Taylor GW (1986) Purification and structural analysis of pyocyanin and 1-hydroxyphenazine. Eur J Biochem 159: 309-313.

Wilson R, Pitt T, Taylor G, Watson D, MacDermot J, Sykes D, Roberts D, Cole P (1987) Pyocyanin and 1-hydroxyphenazine produced by Pseudomonas aeruginosa inhibit the beating of human respiratory cilia in vitro. J Clin Invest 79: 221-229.

Wilson R, Sykes DA, Watson D, Rutman A, Taylor GW, Cole PJ (1988) Measurement of Pseudomonas aeruginosa phenazine pigments in sputum and assessment of their contribution to sputum sol toxicity for respiratory epithelium. Infect Immun 56: 2515-2517.

Zachara BA, Gromadzinska J, Wasowicz W, Zbrog Z (2006) Red blood cell and plasma glutathione peroxidase activities and selenium concentration in patients with chronic kidney disease: a review. Acta Biochim Polon 53: 663-77. 